\title{
Sex differences in flea infections among rodent hosts: is there a male bias?
}

\author{
Krzysztof Kowalski • Michal Bogdziewicz • \\ Urszula Eichert • Leszek Rychlik
}

Received: 16 September 2014 / Accepted: 9 November 2014 / Published online: 21 November 2014

(C) The Author(s) 2014. This article is published with open access at Springerlink.com

\begin{abstract}
Recognizing patterns of parasite distribution among wildlife hosts is of major importance due to growing risk of transmission of zoonotic diseases to humans. Thus, sex-dependent parasite distribution in higher vertebrates is extensively studied, and males are often found more parasitized than females. Male-biased parasitism may be the result of weaker immunocompetence of male hosts owing to the immunosuppressive effect of androgens. Moreover, larger hosts (males) may demonstrate higher parasite infestation levels than smaller individuals (females), as they constitute a better nutritional resource for parasites and provide them with a greater variety of niches. In the present work, we investigated sex-dependent patterns of flea distribution among three common rodent species (Apodemus agrarius, Apodemus flavicollis, and Myodes glareolus). We hypothesized that males have a higher flea infestation than females. We confirm male-biased parasitism in A. agrarius and M. glareolus, but not in A. flavicollis. Additionally, flea infestation increased with body mass in A. agrarius, but not in A. flavicollis and $M$. glareolus. The detected differences in parasite distribution among sexes are probably the result of immunosuppressive effects of androgens and spatial behavior of males.
\end{abstract}

Keywords Sex-biased parasitism - Flea abundance $\cdot$ Body mass $\cdot$ Apodemus agrarius $\cdot$ Apodemus flavicollis $\cdot$ Myodes glareolus

K. Kowalski $(\bowtie) \cdot M$. Bogdziewicz $\cdot$ U. Eichert $\cdot$ L. Rychlik Department of Systematic Zoology, Institute of Environmental Biology, Faculty of Biology, Adam Mickiewicz University, Umultowska 89, 61-614 Poznań, Poland

e-mail: kowalski.biol@gmail.com

\section{Introduction}

Wild rodents are known to be reservoir hosts for many pathogens, which can be transmitted to other animals including humans (Varma and Page 1966; Pawelczyk et al. 2004; Karbowiak et al. 2005a; Klimpel et al. 2007). The most important ectoparasites infesting rodents are fleas and ticks (Pawelczyk et al. 2004) which by transmission of numerous pathogens (e.g., Borrelia ssp., Babesia ssp., Trypanosoma ssp., Leishmania ssp.) can cause serious diseases (Pawelczyk et al. 2004; Karbowiak et al. 2005b; Millán et al. 2014). The importance of rodents as a reservoir of zoonotic diseases is further stressed by their ubiquity, common distribution, and disturbance tolerance (Kryštufek and Griffiths 2002; Bogdziewicz and Zwolak 2014). Due to the high risk of infection in humans, pathogens and their hosts have become a hot spot in public interest (Morand and Poulin 1998; Klimpel et al. 2007; Dziemian et al. 2014).

To allow an accurate description of the pathogen transmission and parasite infestation, extensive research has been conducted on the distribution of fleas in host populations (Morand et al. 2004, Klimpel et al. 2007; Khokhlova et al. 2009a; Krasnov et al. 2011a,b; Kiffner et al. 2013; Kowalski et al. 2014). Evidence has shown that males of higher vertebrates are infested by more parasites than females (Khokhlova et al. 2011; Krasnov et al. 2005, 2011a; Kiffner et al. 2014). In polygynous mating systems, intra-sexual competition favors males that invest more in secondary sexual traits and grow larger. Because of energy constraints, those investments are hypothesized to be done at the expense of immunity (Rolff 2002). Another associated explanation is that in many species males have larger home ranges and often move over longer distances than females (Shenbrot et al. 1997; Stanko et al. 2002, Haapakoski and Ylönen 2010; Krasnov et al. 2011a,b), what may result in encountering more parasitic infectious stages. Furthermore, home range overlaps are usually 
significantly larger for males than for females; this facilitates the horizontal transmission of directly transmitted ectoparasites between males during encounters (Krasnov et al. 2005, 2011a, b).

On the other hand, females of many rodent species usually solitarily nurse their offspring (Clutton-Brock 1991, Lonstein and De Vries 2000, Girard et al. 2002) which facilitates the vertical transmission of ectoparasites between them and juveniles (Yamamura 1993; Ebert and Herre 1996). Moreover, fleas lay eggs in the host nest and therefore female nest may be of higher nutritional value than solitary male nest (Gorrell and Schulte-Hostedde 2008). Indeed, female-biased parasitism has been reported by Krasnov et al. (2005) in several rodent species in South Africa and by Patterson et al. (2008) in 16 neotropical bat species. Thus, despite common, malebiased parasitism is not a universal rule (Kiffner et al. 2013).

It has also been hypothesized that larger hosts (that is males in many/most rodents species) provide a greater variety of niches for parasites and thus can sustain a higher number of parasites (Morand and Poulin 1998; Balashov et al. 2007; Lindenfors et al. 2007). Furthermore, according to the wellfed host hypothesis, they usually represent better nutritional resource for parasites (Christe et al. 2003). Smaller individuals may be also forced to groom more because of the greater surface to volume ratio as the same density of parasites would remove a greater proportion of the hosts' blood (Mooring et al. 2004; Harrison et al. 2010). However, there is still no consensus which of the above mentioned mechanisms is the predominant driver of male-biased parasitism (Gorrell and SchulteHostedde 2008; Harrison et al. 2010; Kiffner et al. 2014).

In the present work, we aimed to test the hypothesis that males of three common rodent species (Apodemus agrarius, Apodemus flavicollis, and Myodes glareolus) have a higher flea infestation than females. To better understand the underlying factors influencing rates, we additionally tested for weight differences between sexes and for differences in infestation in relation to body mass.

\section{Materials and methods}

Study area and trapping procedure

We live-trapped small mammals in the summer of 2010 and 2011 at four distinct localities across Poland (Słowiński National Park, Gorzowska Forest, Konin lakes area, and Bieszczady Mountains). In each, we established two to eight trapping plots and conducted trapping for 2 to 10 consecutive days. Detailed description of study sites and trapping procedure is provided in Kowalski et al. (2014). Captured animals were determined to species and weighed. We recorded also their age, sex, and reproductive activity and marked them individually by ear tagging or cutting a small patch of fur.
Then mammals were placed in a canvas bag for 2-3 min to collect fleas (e.g., Haas and Walton 1973; Paramasvaran et al. 2009; Zuo et al. 2011; Kowalski et al. 2014). Collected fleas were placed in a vial with alcohol and mammals were released at the place of capture.

Data analysis

We tested for differences in body mass between sexes using GLM with log-transformed individual body mass as a response variable and sex and species interaction as fixed factors. To analyze between-sex differences in flea infestation, we used generalized linear mixed models (GLMMs) implemented via the R package lme4 (Bates et al. 2013) and run separate analysis for each investigated species: A. agrarius (model 1), A. flavicollis (model 2), and M. glareolus (model 3). All models were fitted using Poisson family error terms and log link function with trapping site included as a random effect to control for spatial variability in infestation (Kiffner et al. 2013, 2014; Kowalski et al. 2014). In model 2, we detected overdispersion and therefore included additional random effect with unique identifier for each observation (i.e., for each individual sampled); this procedure allows for correction of standard errors and is identical to using overdispersed Poisson model (Zwolak et al. 2013). In each model, we used sex as fixed factor (male vs. female) and individual body mass as a covariate. All models were conducted using data obtained only from adult individuals (only first capture). Moreover, all pregnant and lactating females were removed from these analyses because of confounding effects of pregnancy on body mass. All statistical tests were carried out using R (R Core Team 2014).

\section{Results}

We collected fleas from 69 individuals of A. agrarius, 152 of A. flavicollis, and 105 of M. glareolus. In sum, we collected 488 fleas individuals (70, 198, and 220, from A. agrarius, A. flavicollis, and M. glareolus, respectively) belonging to 11 species. Thirty-four A. agrarius, 79 A. flavicollis, and 36 M. glareolus did not carry any fleas. Maximum flea loads were 6 in A. agrarius, 14 in A. flavicollis, and 13 in M. glareolus.

Males and females did not differ in body mass in our dataset (sex by species effect: $\chi^{2}=0.08, d f=2, p=1.31, p=$ 0.96; Fig. 1). In A. agrarius and $M$. glareolus, fleas were more abundant on males than females (A. agrarius: $\chi^{2}=7.81, d f=1$, $p=0.005$, M. glareolus: $\chi^{2}=8.75, d f=1, p=0.003 ;$ Fig. 2$)$. We found no difference in parasite load between sexes in A. flavicollis $\left(\chi^{2}=0.16, d f=1, p=0.68\right)$. Infestation increased with body mass in $A$. agrarius $\left(z_{1,25}=4.07, p<0.001\right)$ but not 
Fig. 1 Body mass of studied species (A. flavicollis,

A. agrarius, Myodes glareolus). Females $(F)$ are shown with grey and males $(M)$ with white boxes. Boxes denote 25th, 50th, and 75th percentiles; whiskers denote the furthest data points within 1.5 interquartile range

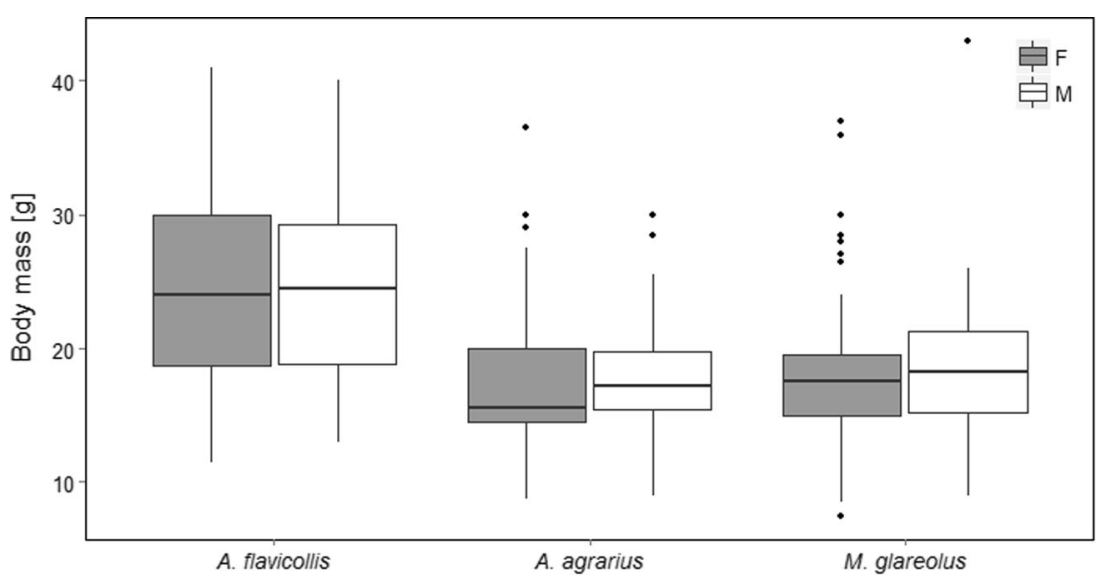

in A. flavicollis $\left(z_{1}, 104=-0.45, p=0.64\right)$ nor in $M$. glareolus $\left(z_{1,33}=-0.01, p=0.98\right)$.

\section{Discussion}

Our results confirm male-biased parasitism in A. agrarius and M. glareolus. However, parasite load did not differ between sexes in A. flavicollis. The detected differences in parasite distribution among sexes are probably the result of immunosuppressive effects of androgens rather than bigger size of males as body mass of sexes did not differ in our dataset (Hillegass et al. 2008; Khokhlova et al. 2009b; Kiffner et al. 2014). Moreover, flea infestation increased with body mass only in A. agrarius, but not in A. flavicollis nor in M. glareolus. Therefore, we can suppose that other factors than body mass, like differences in spatial behavior, movement patterns, and lower immunocompetence of males, may be responsible for found differences (Balashov et al. 2007; Hillegass et al. 2008; Krasnov et al. 2012).

Two main hypotheses regarding male-biased parasitism have been proposed (Khokhlova et al. 2010). The first hypothesis explains male-biased parasitism by the lower immunocompetence of male hosts owing to the immunosuppressive effect of androgens (Zuk and McKean 1996; Harrison et al. 2010; Khokhlova et al. 2011; Krasnov et al. 2011a). Moreover, males may invest less energy in immune responses than females because males engage in intrasexual competition and courtship (Sheridan et al. 2000; Harrison et al. 2010).

The second, not mutually exclusive, hypothesis explains this phenomenon by the higher mobility of males, which facilitates encounters with parasites (e.g., Brown et al. 1994; Khokhlova et al. 2009a; Krasnov et al. 2011a, b, 2012). Males of many rodent species have larger home ranges than females and often move over longer distances than females (Shenbrot et al. 1997; Stanko et al. 2002; Haapakoski and Ylönen 2010; Krasnov et al. 2011a, b). Furthermore, home range overlaps are usually much higher in males than females, which facilitate the horizontal transmission of ectoparasites between males during encounters (Krasnov et al. 2011a, b).

Our data corroborate results obtained by Kiffner et al. (2014), who had reported male-biased parasitism in A. agrarius and M. glareolus. However, contrary to our results, they found effect of body mass on parasite infestation level in M. glareolus. Morand et al. (2004) and Krasnov et al. (2011b) also found a male-biased parasitism in A. agrarius, A. flavicollis, and M. glareolus. However, Krasnov et al. (2011b) found higher flea load in male hosts only in winter.
Fig. 2 Flea abundance on males and females of three studied species (A. flavicollis, A. agrarius, Myodes glareolus). Females $(F)$ are shown with grey and males $(M)$ with white boxes. Boxes denote 25 th, 50 th and 75 th percentiles; whiskers denote the furthest data points within 1.5 interquartile range

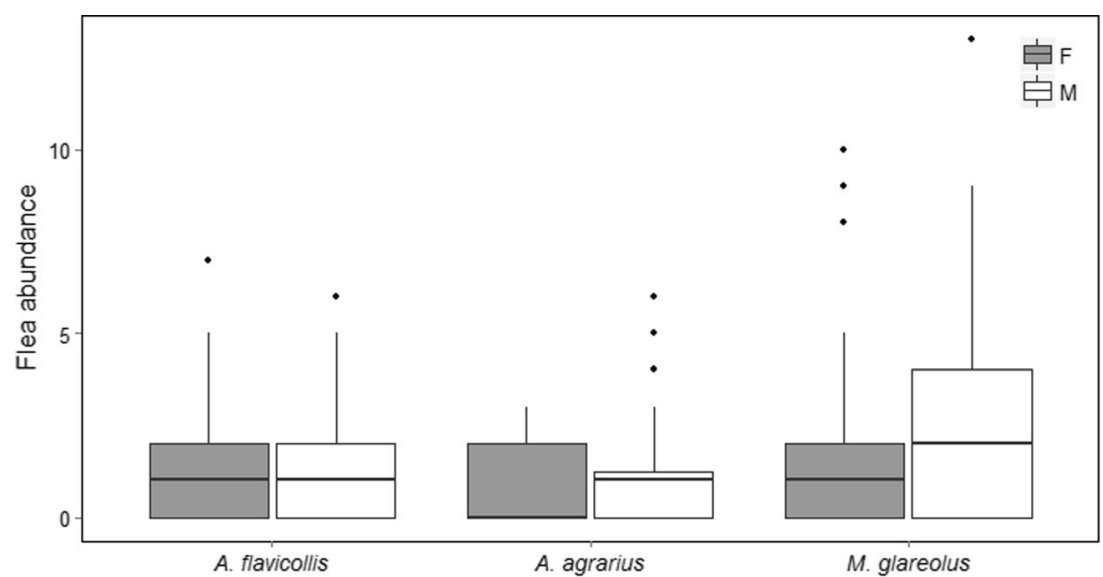


As larger hosts provide a greater variety of niches for parasites and they can sustain higher number of parasites (Morand and Poulin 1998; Balashov et al. 2007), body mass can determine parasite infestation in hosts of different taxa (Stanko et al. 2002; Kiffner et al. 2013, 2014). In addition, according to the well-fed host hypothesis, larger animals should be more parasitized, as they represent a better nutritional resource (Hawlena et al. 2005; Gorrell and SchulteHostedde 2008). Gorrell and Schulte-Hostedde (2008) found that flea infestation was weakly correlated with body size in females of red squirrels, but not in males. In contrary, Harrison et al. (2010) suggest that male-biased parasitism in Apodemus sylvaticus is driven by differences in body size between sexes.

As we found the influence of host body mass on parasite load only in one out of three studied species, we suppose that other factors than body mass are responsible for found differences. Generally, males of all these three rodent species have larger home ranges and move over longer distances than females (Viitala and Hoffmeyer 1985; Vukićević-Radić et al. 2006). However, we found male-biased parasitism only in A. agrarius and M. glareolus, not in A. flavicollis. Presumably, because of lower immunocompetence males represent a more suitable patch for parasites than females (Zuk and McKean 1996; Harrison et al. 2010; Khokhlova et al. 2011; Krasnov et al. 2011a).

Mice of the genus Apodemus are the most widespread small mammals in the temperate zone of the Palearctic region (Ondriková et al. 2010). A. agrarius and A. flavicollis are very common small rodents in Poland and usually co-occur with other rodent species (e.g., M. glareolus). These three species show some preferences towards human settlements and agricultural areas (Ondriková et al. 2010). As pathogens can be transmitted from wild rodents to pets and humans (Karbowiak et al. 2005a; Klimpel et al. 2007), interactions between parasites and their hosts should be a hot spot in public interest. A better understanding of the mechanisms of parasitic infestation in wild animals and the transmission of pathogens to other animals including humans is necessary in an effective prevention of zoonotic diseases. Especially, data on the local prevalence and abundance of parasites of different taxa constitute a basic knowledge to raise public awareness and take a control of parasite infestation in pets and humans (Knaus et al. 2014).

Acknowledgments We are very grateful to A. Stachowiak, P. Kardynia, N. Osten-Sacken, D. Matuszyk, S. Pagacz, J. Witczuk, and W. Eichert for help in field works; to the director of Słowiński National Park for permission to work at the Park; to R. Zwolak and S. DziemianZwolak for access to research material collected in Gorzowska Forest; to B. Piłacińska, and R. Bajaczyk for help in determining rodent and flea species; and to S. Dziemian-Zwolak and S. von Merten for the advice and their comments on the manuscript. The research was supported by the Science and Technology Foundation (Portuguese Ministry of Science, Technology and Higher Education), grant no. SFRH/BD/ 31602/2006 and the budget of the Department of Systematic Zoology (Faculty of Biology AMU, Poznań).
Ethical standards All handling of animals was done with permissions received from the Local Ethical Committee for the Animal Experiments in Poznań, the General Director for Environmental Protection and from the Polish Minister of the Environment.

Conflict of interest The authors declare that they have no conflict of interest.

Open Access This article is distributed under the terms of the Creative Commons Attribution License which permits any use, distribution, and reproduction in any medium, provided the original author(s) and the source are credited.

\section{References}

Balashov YS, Bochkov AV, Vashchenok VS, Grigor'eva LA, Stanyukovich MK, Tret'yakov KA (2007) Segregation of ectoparasitic communities of small forest mammals among ecological niches. Dokl Biol Sci 415:280-283. doi:10.1134/ S0012496607040096

Bates D, Maechler M, Bolker B, Walker S (2013) lme4: linear mixedeffects models using Eigen and S4. R package version 1.0-4. http:// cran.r-project.org/web/packages/lme4/index.html. Accessed December 2013

Bogdziewicz M, Zwolak R (2014) Responses of small mammals to clearcutting in temperate and boreal forests of Europe: a meta-analysis and review. Eur J For Res 133:1-11. doi:10.1007/s10342-013-0726-x

Brown ED, Macdonald DW, Tew TE, Todd IA (1994) Apodemus sylvaticus infected with Heligmosomoides polygyrus (Nematoda) in arable ecosystems: epidemiology and effects of infection on the movement of male mice. J Zool (Lond) 234:623-640. doi:10.1111/j. 1469-7998.1994.tb04869.x

Christe P, Giorgi MS, Vogel P, Arlettaz R (2003) Differential species-specific ectoparasitic mite intensities in two intimately coexisting sibling bat species: resource-mediated host attractiveness or parasite specialization? J Anim Ecol 72:866-872. doi:10.1046/j.1365-2656.2003.00759.x

Clutton-Brock TH (1991) The evolution of parental care. Princeton University Press, Princeton, NJ

Dziemian S, Michalik J, Piłacińska B, Bialik S, Sikora B, Zwolak R (2014) Infestation of urban populations of the Northern whitebreasted hedgehog, Erinaceus roumanicus, by Ixodes spp. ticks in Poland. Med Vet Entomol. doi:10.1111/mve.12065

Ebert D, Herre EA (1996) The evolution of parasitic diseases. Parasitol Today 12:96-101. doi:10.1016/0169-4758(96)80668-5

Girard I, Swallow JG, Carter PA, Koteja P, Rhodes JS, Garland T Jr (2002) Maternal-care behavior and life-history traits in house mice (Mus domesticus) artificially selected for high voluntary wheel-running activity. Behav Process 57:37-50. doi:10.1016/S0376-6357(01)00206-6

Gorrell JC, Schulte-Hostedde AI (2008) Patterns of parasitism and body size in red squirrels (Tamiasciurus hudsonicus). Can J Zool 86:99107. doi:10.1139/Z07-123

Haapakoski M, Ylönen H (2010) Effects of fragmented breeding habitat and resource distribution on behavior and survival of the bank vole (Myodes glareolus). Pop Ecol 52:427-435. doi:10.1007/s10144-010-0193-x

Haas GE, Walton DW (1973) Fleas (Siphonaptera) infesting small mammals from the Western Oriental region. Korean J Parasitol 11:102107

Harrison A, Scantlebury M, Montgomery WI (2010) Body mass and sex biased parasitism in wood mice Apodemus sylvaticus. Oikos 119: 1099-1104. doi:10.1111/j.1600-0706.2009.18072.x

Hawlena H, Abramsky Z, Krasnov BR (2005) Age-biased parasitism and density dependent distribution of fleas (Siphonaptera) on a desert rodent. Oecologia 146:200-208. doi:10.1007/s00442-005-0187-0 
Hillegass MA, Waterman JM, Roth JD (2008) The influence of sex and sociality on parasite loads in an African ground squirrel. Behav Ecol 19:1006-1011. doi:10.1093/beheco/arn70

Karbowiak G, Rychlik L, Nowakowski W, Wita I (2005a) Natural infections of small mammals with blood parasites on the borderland of boreal and temperate forest zones. Acta Theriol 50:31-42. doi:10. 1007/BF03192616

Karbowiak G, Wita I, Rychlik L (2005b) Trypanosoma (Megatrypanum) ornata sp. n., a parasite of the Eurasian water shrew Neomys fodiens (Pennant, 1771). Acta Parasitol 44:363-367

Khokhlova IS, Serobyan V, Degen AA, Krasnov BR (2010) Host gender and offspring quality in flea parasitic on a rodent. J Exp Biol 213: 3299-3304. doi:10.1242/jeb.046565

Khokhlova IS, Serobyan V, Degen AA, Krasnov BR (2011) Discrimination of host sex by a haematophagous ectoparasite. Anim Behav 81:275-281. doi:10.1016/j.anbehav.2010.10.018

Khokhlova IS, Serobyan V, Krasnov BR, Degen AA (2009a) Effect of host gender on blood digestion in fleas: mediating role of environment. Parasitol Res 105:1667-1673. doi:10.1007/s00436-009-1608-5

Khokhlova IS, Serobyan V, Krasnov BR, Degen AA (2009b) Is the feeding and reproductive performance of the flea, Xenopsylla ramesis, affected by the gender of its rodent host, Meriones crassus? J Exp Biol 212:1429-1435. doi:10.1242/jeb.029389

Kiffner C, Stanko M, Morand S, Khokhlova IS, Shenbrot GI, Laudisoit A, Leirs H, Hawlena H, Krasnov BR (2013) Sex-biased parasitism is not universal: evidence from rodent-flea associations from three biomes. Oecologia 173:1009-1022. doi:10.1007/s00442-013-2664-1

Kiffner C, Stanko M, Morand S, Khokhlova IS, Shenbrot GI, Laudisoit A, Leirs H, Hawlena H, Krasnov BR (2014) Variable effects of host characteristics on species richness of flea infracommunities in rodents from three continents. Parasitol Res 113:2777-1788. doi:10. 1007/s00436-014-3937-2

Klimpel S, Förster M, Schmahl G (2007) Parasites of two abundant sympatric rodent species in relation to host phylogeny and ecology. Parasitol Res 100:867-875. doi:10.1007/s00436-006-0368-8

Knaus M, Rapti D, Shukullari E, Kusi I, Postoli R, Xhaxhiu D, Silaghi C, Hamel D, Visser M, Winter R, Rehbein S (2014) Characterisation of ecto- and endoparasites in domestic cats from Tirana, Albania. Parasitol Res 113:3361-3371. doi:10.1007/s00436-014-3999-1

Kowalski K, Eichert U, Bogdziewicz M, Rychlik L (2014) Differentiation of flea communities infesting small mammals across selected habitats of the Baltic coast, central lowlands, and southern mountains of Poland. Parasitol Res 113:1725-1734. doi:10.1007/s00436-014-3817-9

Krasnov BR, Bordes F, Khokhlova IS, Morand S (2012) Gender-biased parasitism in small mammals: patterns, mechanisms, consequences. Mammalia 76:1-13. doi:10.1515/mammalia-2011-0108

Krasnov BR, Morand S, Hawlena H, Khokhlova IS, Shenbrot GI (2005) Sex-biased parasitism, seasonality and sexual size dimorphism in desert rodents. Oecologia 146:209-2017. doi:10.1007/s00442-005-0189-y

Krasnov BR, Stanko M, Matthee S, Laudisoit A, Leirs H, Khokhlova IS, Korallo-Vinarskaya NP, Vinarski MV, Morand S (2011a) Male hosts drive infracommunity structure of ectoparasites. Oecologia 166: 109-1110. doi:10.1007/s00442-01-1950-Z

Krasnov BR, Stanko M, Morand S (2011b) An attempt to use ectoparasites as tags for habitat occupancy by small mammalian hosts in central Europe: effects of host gender, parasite taxon and season. Parasitology 138:609-618. doi:10.1017/S0031182011000102

Kryštufek B, Griffiths HI (2002) Species richness and rarity in European rodents. Ecography 25:120-128. doi:10.1034/j.1600-0587.2002.250114.x

Lindenfors P, Nunn CL, Jones KE, Cunningham AA, Sechrest W, Gittleman JL (2007) Parasite species richness in carnivores: effects of host body mass, latitude, geographical range and population density. Global Ecol Biogeographica 16:496-509. doi:10.1111/j.1466-8238.2006.00301.x

Lonstein JS, De Vries GJ (2000) Sex differences in the parental behavior of rodents. Neurosci Biobehav Rev 24:669-686. doi:10.1016/ S0149-7634(00)00036-1
Millán J, Ferroglio E, Solano-Gallego L (2014) Role of wildlife in the epidemiology of Leishmania infantum infection in Europe. Parasitol Res 113:2005-2014. doi:10.1007/s00436-014-3929-2

Mooring MS, Blumstein DT, Stoner CJ (2004) The evolution of parasitedefence grooming in ungulates. Biol J Linnean Soc 81:17-37. doi: 10.1111/j.1095-8312.2004.00273.x

Morand S, Gouy De Belloc J, Stanko M, Miklisova D (2004) Is sexbiased ectoparasitism related to sexual size dimorphism in small mammals of Central Europe? Parasitology 129:505-510. doi:10. $1017 / \mathrm{S} 0031182004005840$

Morand S, Poulin R (1998) Density, body mass and parasite species richness of terrestrial mammals. Evol Ecol 12:717-727. doi:10. 1023/A:1006537600093

Ondriková J, Miklisová D, Ribas A, Stanko M (2010) The helminth parasites of two sympatric species of the genus Apodemus (Rodentia, Muridae) from south-eastern Slovakia. Acta Parasitol 55:369-378. doi:10.2478/s11686-010-0043-1

Paramasvaran S, Sani RA, Hassan L, Krishnasamy M, Jeffery J, Oothuman P, Salleh I, Lim KH, Sumarni MG, Santhana RL (2009) Ectoparasite fauna of rodents and shrews from habitats in Kuala Lumpur and the states of Selangor and Negeri Sembilan, Malaysia and its public health significance. Trop Biomed 26:303-311

Patterson BD, Dick CW, Dittmar K (2008) Sex biases in parasitism of Neotropical bats by bat flies (Diptera: Streblidae). J Trop Ecol 24: 387-396. doi:10.1017/S0266467408005117

Pawelczyk A, Bajer A, Behnke JM, Gilbert FS, Siński E (2004) Factors affecting the component community structure of haemoparasites in common voles (Microtus arvalis) from the Mazury Lake District region of Poland. Parasitol Res 92:270-284. doi:10.1007/s00436-003-1040-1

R Core Team (2014) R: a language and environment for statistical computing. R Fundation for Statistical Computing, Vienna, Austria. http://www.R-project.org. Accessed Oct 2014

Rolff J (2002) Bateman's principle and immunity. Proc R Soc B 269:867872. doi:10.1098/rspb.2002.1959

Shenbrot GI, Krasnov BR, Khokhlova IS (1997) On the biology of Wagner's gerbil (Gerbillus dasyurus Wagner, 1842) (Rodentia: Gerbillidae) in the Negev Highlands, Israel. Mammalia 61:467-486

Sheridan LAD, Poulin R, Ward DF, Zuk M (2000) Sex differences in parasitic infections among arthropod hosts: is there a male bias? Oikos 88:327-334. doi:10.1034/j.1600-0706.2000.880211.x

Stanko M, Miklisová D, Gouy de Bellocq J, Morand S (2002) Mammal density and patterns of ectoparasite species richness and abundance. Oecologia 131:289-295. doi:10.1007/s00442-00200889-5

Varma MGR, Page RJC (1966) The epidemiology of louping ill in Ayrshire, Scotland: ectoparasites of small mammals. I. (Siphonaptera). J Med Entomol 3:331-335

Viitala J, Hoffmeyer I (1985) Social organization in Clethrionomys compared with Microtus and Apodemus: social odours, chemistry and biological effects. Ann Zool Fenn 22:359-371

Vukićević-Radić O, Matić R, Kataranovski D, Stamenković S (2006) Spatial organization and home range of Apodemus flavicollis and A. agrarius on Mt. Avala, Serbia. Acta Zool Hung 52:81-96

Zuk M, McKean KA (1996) Sex differences in parasite infections: patterns and processes. Int J Parasitol 26:1009-1024. doi:10.1016/ S0020-7519(96)80001-4

Zuo XH, Guo XG, Zhan YZ, Wu D, Yang ZH, Dong WG, Huang LQ, Ren TG, Jing YG, Wang QH, Sun XM, Lin SJ (2011) Host selection and niche differentiation in sucking lice (Incecta: Anoplura) among small mammals in southwestern China. Parasitol Res 108:12431251. doi: $10.1007 / \mathrm{s} 00436-010-2173-7$

Zwolak R, Meagher S, Vaughn JW, Dziemian S, Crone EE (2013) Reduced ectoparasite loads of deer mice in burned forest: from fleas to trees? Ecosphere. doi:10.1890/ES13-00138.1, 4:art132

Yamamura N (1993) Vertical transmission and evolution of mutualism from parasitism. Theor Popul Biol 44:95-109. doi:10.1006/tpbi. 1993.1020 